\title{
Acknowledgement of reviewers 2012
}

Published online: 1 November 2013

(C) Springer Science+Business Media New York 2013

The journal editors are grateful to the following reviewers for their dedicated service in 2012 .

Amar Agha

Gianluca Aimaretti

Olaf Ansorge

Emanuela Arvat

Simon Aylwin

John Ayuk

Indi Banerjee

G. Baumann

Anat Ben-Shlomo

Ronald Benveniste

Antonio Bianchi

Nienke Biermasz

Marco Boscaro

Georg Brabant

Karin Bradley

M. D. Bronstein

O. D. Bruno

Michael Buchfelder

Pia Burman

Paolo Cappabianca

John Carmichael

P. Caron

Francesco Cavagnini

Harvinder Chahal

William Chandler

Vera Chesnokova

Richard Clayton

William T. Couldwell

Simon Cudlip

Adrian Daly

Christina Daousi
Mehul Dattani

Julian Davis

Annamaria De Bellis

Laura de Marinis

Ernesto de Menis

Ettore degli Uberti

Olaf Dekkers

Eleni Dimaraki

Neil Dorward

Will Drake

Pinaki Dutta

Sarah Ehtisham

Cihangir Erem

Eva Erfurth

Shereen Ezzat

P. Fainstein Day

William Farrell

Richard Feelders

Hugo L. Fideleff

Prodam Flavia

Maria Fleseriu

Pamela Freda

Monica Gadelha

Federico Gatto

Stephen Gebarski

Mary P. Gillam

Neil Gittoes

Andrea Giustina

Monica Gola

Y. Greenman

Mirtha Guitelman
Amir Hamrahian

Anthony Heaney

Andrew Hoffman

Ian Holdaway

Juergen Honegger

Wenyu Huang

Gila Idelman

John A. Jane

Paul Jenkins

Jens Jorgensen

Niki Karavitaki

Marleen Kars

Laurence Katznelson

Fahrettin Kelestimur

Sibylle Kohler

George Kontogeorgos

Marta Korbonits

K. Kovacs

Hau Kwaan

Ed Laws

Paola Loli

Marco Losa

Malcolm Low

D. Lunsford

Mohamad Maghnie

Adam Mamelak

Ann Mccormack

Moises Mercado

Nobuhiro Miki

Giuseppe Minniti

Mark Molitch 
Pietro Mortini

Robert D. Murray

Lisa Nachtigall

Sebastian Neggers

John Orrego

Leena Patel

Alberto Pereira

Vera Popovic

\section{Kalmon Post}

Michael Powell

R. Quinton

M. Racine

Ferdinand Roelfsema

R. J. M. Ross

Fabio Rotondo

Fiona Ryan

\section{Roberto Salvatori}

Carla Scaroni

Omar Serri

Gufta Mohamad Shaikh

Mark Sherlock

Ilan Shimon

Thomas Smith

Nicoletta Sonino

Stephen Sullivan

B. Swearingen

Francesca Swords

Massimo Terzolo

Nick Thomas

Chris Thompson

Peter Trainer

Nicholas Tritos
Giovanni Tulipano

Elena Valassi

Mary Lee Vance

Joseph Verbalis

Jose Vieira

S. M. Webb

Jonathan Webster

Run Yu

Kevin Yuen

Maria Chiara Zatelli

Xun Zhang

* Those in bold submitted 6 or more reviews to the journal in 2012 